\title{
Differential long non-coding RNA expression profile and function analysis in primary Sjogren's syndrome
}

\author{
Xiaochan Chen, Qi Cheng, Yan Du, Lei Liu and Huaxiang Wu*
}

\begin{abstract}
Background: Primary Sjögren's syndrome (pSS) is a chronic autoimmune disease characterized by abnormal immune cell activation. This study aimed to investigate differentially expressed long non-coding RNA (IncRNA) in peripheral blood mononuclear cells (PBMCs) in patients with pSS to identify IncRNAs that affect pSS pathogenesis.

Methods: Total RNA was extrated from PBMCs of 30 patients with pSS and 15 healthy persons. Transcriptome sequencing was used to screen differentially expressed IncRNAs and mRNAs in 8 RNA samples from the discovery cohort. The differentially expressed mRNAs underwent functional enrichment analysis. A protein interaction relationship (PPI) and competitive endogenous RNA (ceRNA) network was constructed. Real-time PCR was used to validate screened IncRNAs in all 45 RNA samples..

Results: 1180 IncRNAs and 640 mRNAs were differentially expressed in pSS patients (fold change $>2$ in healthy persons). The PPI network was constructed with 640 mRNAs and a ceRNA network with four key IncRNAs (GABPB1AS1, PSMA3-AS1, LINC00847 and SNHG1). Real-time PCR revealed that GABPB1-AS1 and PSMA3-AS1 were significantly up-regulated 3.0- and 1.4-fold in the pSS group, respectively. The GABPB1-AS1 expression level was positively correlated with the percentage of B cells and lgG levels.

Conclusions: GABPB1-AS1 was significently up-regulated in pSS patients, and its expression level is positively correlated with the percentage of B cells and IgG levels. GABPB1-AS1 may be involved in the pathogenesis of pSS and may be a promising biological marker.
\end{abstract}

Keywords: LncRNA, GABPB1-AS1, Sjogren's syndrome, PBMC

\section{Background}

Primary Sjogren's syndrome (pSS) is a chronic autoimmune disease which clinically presents with endocrine gland dysfunction, mainly occurring in the salivary and lacrimal glands. Histopathology typically shows infiltration of lymphocytes. In particular, large amounts of activated B lymphocytes are observed, which produce a variety of autoantibodies and cytokines; this is related to the occurrence and development of the disease.

\footnotetext{
* Correspondence: wuhx8855@zju.edu.cn

Department of Rheumatology, The Second Affiliated Hospital of Zhejiang University School of Medicine, No.88 Jiefang Road, Hangzhou 310009, China
}

Although multiple factors such as genetic and environmental factors are thought to be related to this abnormal cellular activity, the specific mechanisms of pSS have not been fully elucidated.

Non-coding RNAs are RNA sequences in the human genome that cannot encode proteins and are largely categorized into short-chain non-coding RNAs (microRNAs, miRNAs) (<200 nt) and long-chain non-coding RNAs (lncRNAs) (>200 nt). A large number of studies have shown that miRNAs participate in regulating the pathogenesis of some autoimmune diseases due to their control of gene expression [1, 2]. Moreover, miRNAs

(c) The Author(s). 2021 Open Access This article is licensed under a Creative Commons Attribution 4.0 International License, which permits use, sharing, adaptation, distribution and reproduction in any medium or format, as long as you give appropriate credit to the original author(s) and the source, provide a link to the Creative Commons licence, and indicate if changes were made. The images or other third party material in this article are included in the article's Creative Commons licence, unless indicated otherwise in a credit line to the material. If material is not included in the article's Creative Commons licence and your intended use is not permitted by statutory regulation or exceeds the permitted use, you will need to obtain permission directly from the copyright holder. To view a copy of this licence, visit http://creativecommons.org/licenses/by/4.0/ The Creative Commons Public Domain Dedication waiver (http://creativecommons.org/publicdomain/zero/1.0/) applies to the data made available in this article, unless otherwise stated in a credit line to the data. 
affect the development of Sjögren's syndrome [3]. Unlike miRNAs, the functions of most lncRNAs are largely unknown because their structure and regulatory patterns are more complex than miRNAs. The known lncRNAs appear to exert a regulatory role in gene transcription or expression and function as scaffold molecules to regulate the expression of certain miRNA target genes [4]. Therefore, lncRNAs may affect the development of autoimmune diseases by regulating gene expression during immune cell differentiation and immune responses.

Some preliminary studies have found the role of lncRNAs in regulating gene expression, consequently affecting the pathogenesis of immune diseases. For example, a study showed that lncRNA NeST affects microbial susceptibility by increasing interferon- $\gamma$ (IFN- $\gamma$ ) levels in activated CD8(+) $\mathrm{T}$ cells [5]. lncRNA NEAT1 regulates chemokine and cytokine expression and contributes to the progression of systemic lupus erythematosus [6]. IncRNA Hotair expression in differentiated osteoclasts and rheumatoid synovial cells is closely related to a significant increase in MMP-2 and MMP-13 levels, suggesting that lncRNA Hotair participates in rheumatoid arthritis by promoting matrix metalloproteinase (MMP) production [7]. IncRNA FIRRE regulates the expression of VCAM1, IL-12p40, and other inflammatory genes in the innate immune system at the posttranscriptional level [8]. It can promote $\mathrm{B}$ cell proliferation by activating the $\mathrm{Wnt} / \beta$-catenin pathway and it is related to the pathogenesis of diffuse large $B$ cell lymphoma [9]. Based on the previous studies, lncRNAs are expected to be biomarkers for disease diagnosis or therapeutic targets.

Recently, two researches have focused on the expression profile of LncRNAs in peripheral blood mononuclear cells (PBMCs) of pSS patients. Peng et al. found two significantly up-regulated lncRNAs (NRIR and BISPR) and discovered they may regulate important genes involved in the Type I interferon signaling pathway [10]. Dolcino et al. observed six DE lncRNAs (CTD2020 K17.1, LINC00657, RP11-169 K16.9, LINC00511, RP11-372 K14.2 and RP11-214O1.2) and found they target gene pathways that are involved in epithelial cell damage, autoimmunity and B cell hyperactivation [11]. To date, however, the correlation between IncRNAs and pSS has not been well studied.

In order to provide more reliable evidences, this study analyzed lncRNA and mRNA expression profiles in PBMCs of patients with pSS using transcription sequencing and then identified lncRNAs related to pSS using real-time PCR in a relatively large cohort. Our findings provide a new perspective for the pathogenesis study and identified potential disease-related biological markers and therapeutic targets for pSS.

\section{Result}

\section{Patient characteristics}

The characteristics of the $30 \mathrm{pSS}$ patients are shown in Table 1 . The enrolled patients were positive for antinuclear antibodies $(\geq 1: 80)$, in which 25 and 8 patients were positive for anti-SSA/Ro antibodies and anti-SSB/La antibodies, respectively. Ocular and oral dryness were reported in approximately 20 and 24 patients, respectively. Lymphocyte infiltration was found in 25 patients who underwent labial salivary gland biopsies. The median Eular Sjogren's syndrome disease activity index (ESSD AI) score was 3.8 , the median B cell/lymphocyte was $13.4 \%$, and the mean $\operatorname{IgG}$ was $16.3 \pm 4.3$. g/L. B cell lymphoma occurred in one patient after 3 months in the study.

\section{High-throughput IncRNA and mRNA expression profile in PBMC}

We found 1180 lncRNAs and 640 mRNAs with significantly different expression in the PBMC of pSS patients based on transcriptome sequencing (fold change $>2, p<$ 0.05). The up-regulated genes included 497 lncRNAs and 256 mRNAs. The down-regulated genes included 683 lncRNAs and 384 mRNAs. The differentially expressed (DE) genes are displayed using a volcanic map (Fig. 1a) and heatmap (Fig. 1b). Tables 2 and 3 list the 15 most up-regulated and down-regulated DE lncRNAs and DE mRNAs.

\section{Functional prediction and protein interaction relationship (PPI) network of DE mRNAs}

The 640 DE mRNAs were subjected to GO and KEGG pathway detection to determine the correlation of signaling pathways to pSS pathological mechanisms. Table 4 lists the top 30 significant GO enrichment of differentially expressed mRNAs. Significantly enriched biological processes known to affect pSS include immune response, chemokine-mediated signaling pathway, cell adhesion, type I interferon signaling pathway, and inflammatory response. Significantly enriched cellular components include the plasma membrane, extracellular region, integral component of membrane, and extracellular exosome. Significantly enriched molecular functions include calcium ion binding, protein homodimerization activity, receptor activity, and heparin binding. Significantly enriched pathways include chemokine signaling pathway, PI3K-Akt signaling pathway, transcriptional misregulation in cancer, natural killer cell-mediated cytotoxicity, and cytokine-cytokine receptor interaction (Table 5).

According to the description in the Methods section, we obtained 2033 protein interaction pairs in $488 \mathrm{DE}$ mRNAs, including 201 up-regulated genes and 287 down-regulated genes. The PPI network of $640 \mathrm{DE}$ 
Table 1 The detailed demographic, clinical, and laboratory characteristics of 30 pSS patients

\begin{tabular}{lll}
\hline Indexes & RNA sequencing $(\boldsymbol{n}=\mathbf{4})$ & PCR validation $(\boldsymbol{n}=\mathbf{3 0})$ \\
\hline Sex, no. Male/female & $0 / 4$ & $0 / 30$ \\
Age, mean (SD) years & $47.0(12.1)$ & $52.7(13.1)$ \\
Disease duration, median (IQR) years & $3.5(1.0)$ & $1.0(6.2)$ \\
Oral dryness (VAS,1-10), median (IQR) & $5.0(2.0)$ & $5.5(4.3)$ \\
Ocular dryness (VAS,1-10), median (IQR) & $3.5(1.0)$ & $3.0(5.3)$ \\
Grading of labial salivary gland biopsies, no. & & 1 \\
$\quad$ Grade2, no. & 0 & 11 \\
Grade3, no. & 2 & 13 \\
$\quad$ Grade4, no. & 2 & $17.5(3.4)$ \\
IgG (g/L), mean \pm SD & $14.9(2.5)$ & $0.83(0.24)$ \\
C3 (g/L), mean $\pm S D$ & $0.89(0.24)$ & $156.8(72.6)$ \\
C4 (g/L), mean $\pm S D$ & $154.3(48.1)$ & $30(45)$ \\
ESR (mm/h), mean $\pm S D$ & $25(19)$ & $12.7(19.8)$ \\
RF (IU/mL), mean $\pm S D$ & $12.4(2.7)$ & $25 / 5$ \\
SSA, no. +/- & $4 / 0$ & $8 / 22$ \\
SSB, no. +/- & $1 / 3$ & $18.2(7.8)$ \\
B cell, \%. median (IQR) & $23.1(8.3)$ & $3.8(1.9)$ \\
ESSDAI score, median (IQR) & $2.5(1.0)$ &
\end{tabular}

$S D$ standard deviation, IQR inter-quartile range, VAS visual analogue scale, IgG immunoglobulin $\mathrm{G}, C 3 / C 4$ complement $3 / 4, E S R$ erythrocyte sedimentation rate, $R F$ rheumatoid factor, ESSDAl Eular Sjogren's syndrome disease activity index

mRNAs was built (Fig. 2a), and two important functional modules were obtained. Table 6 shows the top 10 genes which resulted from the network connectivity analysis, among which IL-6, IL-10, and CXCL8 are at the center of the network and possibly the hub genes in the PPI network. Through module analysis, we obtained two important functional modules (Fig. $2 \mathrm{~b}$ and $\mathrm{c}$ ). Module 1 contains 30 genes and 292 interaction pairs, while module 2 contains 33 genes and 266 interaction pairs. The pathways that the genes in module 1 are involved in include the calcium signaling pathway, cytokine-cytokine receptor interaction, and chemokine signaling pathway (Fig. 2d). The pathways that the genes in module 2 are involved in include natural killer cell-mediated cytotoxicity, cytokine-cytokine receptor interaction, and JAKSTAT signaling pathway (Fig. 2e). These genes and pathways are known to influence the pathogenesis of pSS.

\section{Competitive endogenous RNA (ceRNA) network of four selected IncRNAs}

The regulation network of the top 15 up-regulation and down-regulation DE IncRNAs predicted by Trans and Cis regulation was constructed (Fig. 3a). Among them, GABPB1-AS1 regulates 187 genes, including two upregulated genes (TRPM4 and SPATS2L) and two downregulated genes (SCUBE1, CTD-2192 J16.20). To verify whether the DE IncRNAs were involved in the pathogenesis of pSS via interaction with miRNA, the top 15 of up-regulated and down-regulated DE IncRNAs were selected to construct the ceRNA network based on starBase. Only experimentally validated miRNA or mRNA targets annotated in the software were retained. Four lncRNAs, including GABPB1-AS1, PSMA3-AS1, LINC00847, and SNHG1, were obtained and their ceRNA network is shown in Fig. 3b. In this ceRNA network, there are 17 pairs of IncRNA-miRNA regulating relationships, 11 pairs of IncRNA-mRNA regulating relationships, and 155 pairs of miRNA-mRNA regulating relationships. Co-expression analysis was made between 4 key lncRNAs and 15 most up-regulated and downregulated DE mRNAs (Table 7). Some of these significantly up-regulated mRNAs (IFI44, IFI44L, IFI6, EPST I1) have been identified to be involved in the pathogenesis of pSS according to previous literatures [12-14]. Figure 4 shows the co-expression relationship between these hob genes and 2 up-regulated key lncRNAs (GABPB1-AS1, PSMA3-AS1).

\section{Validation with real-time PCR and function analysis}

Two differentially expressed lncRNAs were chosen for further validation in an independent cohort including 30 patients with pSS and 15 healthy controls. The selected IncRNAs and their primers are presented in Table S2. The results demonstrate that GABPB1-AS1 and PSMA3-AS1 are significantly up-regulated 3.0- and 1.4fold, respectively, in the pSS group compared to the 
a

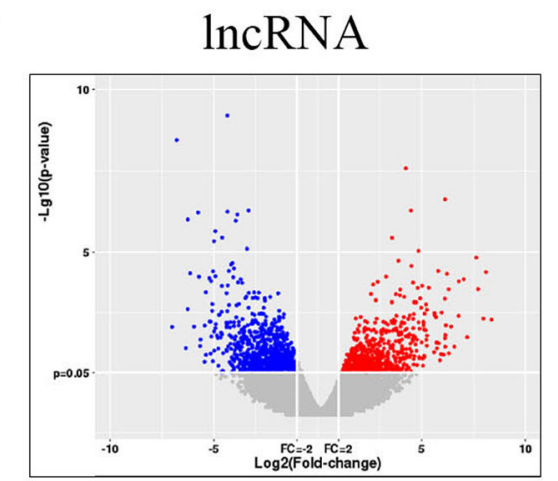

b

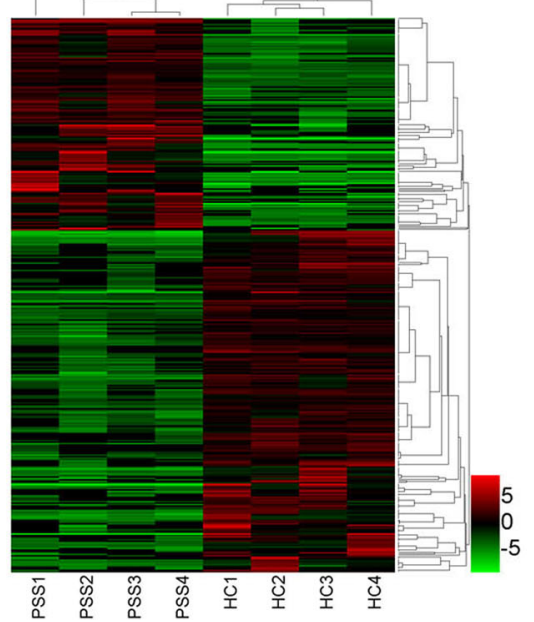

mRNA
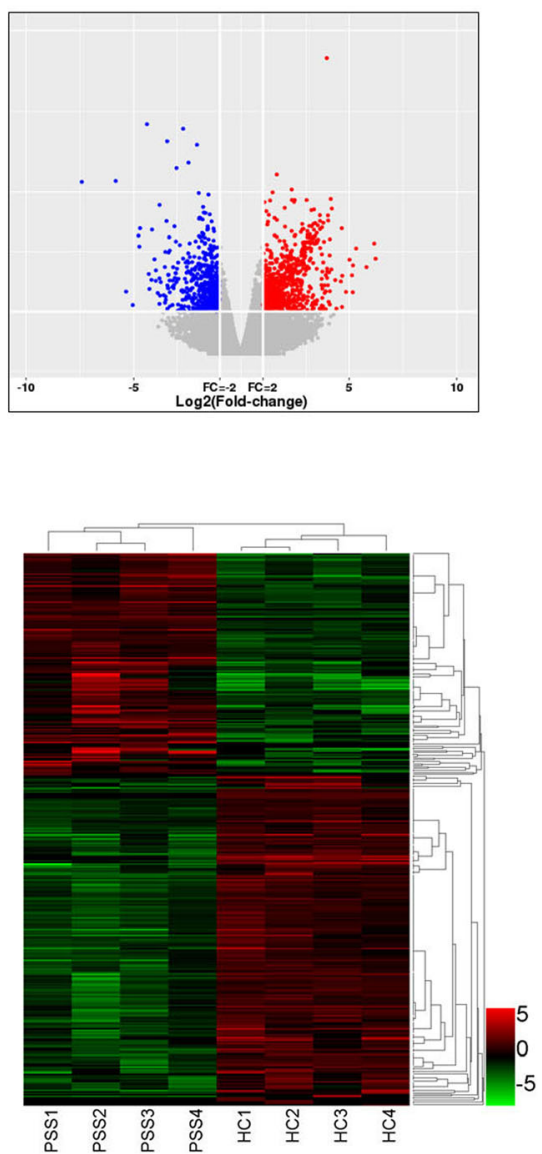

Fig. 1 Expression profile of LnCRNAs and mRNAs in pSS patients. PBMCs of pSS patients and healthy persons were collected for transcriptome sequencing analysis. The differentially expressed IncRNAs and mRNAs were illustrated using (a) volcanic map and (b) heatmap. Up-regulation is represented in red. Down-regulation is represented in blue in the volcanic map and green in the heatmap

Table 2 Top 15 significantly differential expressed IncRNAs between pSS patients and control subjects

\begin{tabular}{|c|c|c|c|c|c|}
\hline up-regulated IncRNA & $P$ value & Fold change & down-regulated IncRNA & $P$ value & Fold change \\
\hline GABPB1-AS1 & 1.36E-09 & 738.802 & RP11-343H5.4 & $2.17 \mathrm{E}-14$ & -856.93 \\
\hline Inc-AC012652.1.1-1-11 & 1.36E-05 & 289.846 & m121218_154352_00126_c100 & $1.18 \mathrm{E}-06$ & -30.374 \\
\hline Inc-TMEM65-4-1 & $1.71 \mathrm{E}-08$ & 51.288 & brain_Pred31224 & 2.85E-08 & -26.599 \\
\hline Inc-RBPMS2-3-1_dup1 & 4.34E-04 & 31.393 & Inc-CNOT7-2-1 & $3.41 \mathrm{E}-04$ & -10.070 \\
\hline m121212_224522_00126_c100 & 1.15E-08 & 18.981 & Inc-ZNF507-1-1_dup1 & $9.88 \mathrm{E}-05$ & -8.296 \\
\hline Inc-YDJC-1-2_dup1 & 3.99E-03 & 8.583 & LINC00847 & 8.57E-03 & -7.030 \\
\hline m130128_030356_00126_c100 & $1.81 \mathrm{E}-03$ & 8.125 & m121218_132759_00126_c100 & $2.00 \mathrm{E}-06$ & -6.950 \\
\hline NRIR & 2.07E-03 & 7.079 & Inc-MYOM2-7-1_dup1 & 3.69E-04 & -5.980 \\
\hline PSMA3-AS1 & 4.37E-04 & 5.963 & m121218_180114_00126_c100 & 3.61E-03 & -5.788 \\
\hline Inc-ZNF503-AS2-4-1 & $5.88 \mathrm{E}-03$ & 4.110 & CUFF.400492 & $1.25 \mathrm{E}-03$ & -5.056 \\
\hline Inc-C3orf67-3-1_dup1 & $6.69 \mathrm{E}-03$ & 3.961 & Inc-SART3-2-1_dup1 & 2.37E-03 & -4.750 \\
\hline Inc-STRBP-9-1 & 8.99E-03 & 3.689 & brain_Pred8666 & $7.42 \mathrm{E}-03$ & -4.069 \\
\hline RPL32P1 & $7.02 \mathrm{E}-04$ & 3.567 & SNHG1 & 2.31E-04 & -3.550 \\
\hline Inc-RNF150-2-4 & $1.25 \mathrm{E}-02$ & 3.496 & Inc-CLEC4C-1-1_dup1 & $2.86 \mathrm{E}-03$ & -3.498 \\
\hline Inc-ERMN-2-1_dup1 & 8.88E-03 & 3.104 & EBLN3 & $6.98 \mathrm{E}-03$ & -3.423 \\
\hline
\end{tabular}


Table 3 Top 15 significantly differential expressed mRNAs between pSS patients and control subjects

\begin{tabular}{lllllll}
\hline up-regulated $\boldsymbol{m R N A}$ & $\boldsymbol{P}$ value & Fold change & down-regulated mRNA & $\boldsymbol{P}$ value & Fold change \\
\hline MPO & $7.03 \mathrm{E}-05$ & 7.779 & MYOM2 & $1.68 \mathrm{E}-07$ & $1.72 \mathrm{E}-06$ & -9.794 \\
SIGLEC1 & $7.42 \mathrm{E}-08$ & 5.642 & SH2D1B & $1.68 \mathrm{E}-06$ & -4.162 \\
IFI44L & $1.16 \mathrm{E}-06$ & 5.570 & COL6A2 & $8.09 \mathrm{E}-06$ & -3.160 \\
IFI44 & $9.43 \mathrm{E}-08$ & 3.296 & KIR2DL3 & $3.29 \mathrm{E}-08$ & -3.629 \\
IFI6 & $1.45 \mathrm{E}-06$ & 2.868 & ARMCX3 & $2.63 \mathrm{E}-05$ & -3.585 \\
PCNP & $1.96 \mathrm{E}-06$ & 2.840 & KLRF1 & $6.24 \mathrm{E}-05$ & -3.580 \\
CMPK2 & $3.75 \mathrm{E}-05$ & 2.590 & GNLY & $1.75 \mathrm{E}-05$ & -3.406 \\
EPSTI1 & $1.42 \mathrm{E}-07$ & 2.559 & PRF1 & $8.96 \mathrm{E}-06$ & -3.370 \\
CD83 & $3.75 \mathrm{E}-07$ & 2.557 & APEX1 & $8.17 \mathrm{E}-05$ & -3.291 \\
ZNF267 & $1.02 \mathrm{E}-05$ & 2.525 & SPON2 & $1.15 \mathrm{E}-04$ & -3.192 \\
PIM3 & $1.12 \mathrm{E}-04$ & 2.460 & FGFBP2 & $1.13 \mathrm{E}-05$ & -2.793 \\
U2AF1 & $3.65 \mathrm{E}-05$ & 2.313 & KLRB1 & $9.46 \mathrm{E}-05$ & -2.754 \\
SPI1 & $9.36 \mathrm{E}-06$ & 2.290 & CPA3 & $4.00 \mathrm{E}-05$ & -2.471 \\
PLSCR1 & 2.282 & IL2RB & $5.30 \mathrm{E}-05$ & -2.301 \\
LAP3 & $2.90 \mathrm{E}-06$ & LDLRAP1 & &
\end{tabular}

healthy control (Fig. 5a). We further investigated the correlation of the two lncRNAs with clinical characteristics in patients with pSS and health controls. We found that the expression level of GABPB1-AS1 is positively correlated with the percentage of B cells and IgG levels (Fig. 5b). While the expression level of PSMA3-AS1 has no significant correlation with the percentage of B cells and IgG levels (Fig. 5c). Unfortunately, other clinical characteristics such as complement C3/C4, ESR, RF, SSA, and ESSDAI were not found any correlation with the expression level of GABPB1-AS1 or PASM3-AS1 (Table S3).

\section{Discussion}

Recent evidence has demonstrated the essential role of lncRNAs in various types of autoimmune diseases including pSS due to their control of gene expression at multiple levels [15]. Shi et al. revealed eight significantly up-regulated lncRNAs in labial salivary glands in pSS patients and observed correlation between these lncRNAs and some clinical characteristics [16]. LncRNA TMEV PG1 and IncRNA PVT1 were found in another two studies to be up-regulated in CD4(+) T cells of pSS patients and were suggested to be involved in the pathogenesis of pSS [17]. However, the potential role of lncRNAs in pSS is still far from known. In this study, we payed attention to the expression profile of lncRNAs in PBMCs. We screened out DE mRNAs and DE IncRNAs that may play a role in pSS, constructed their regulatory networks, and attempted to identify the key lncRNAs that affect the pathogenesis of pSS.

In this study, GO and KEGG pathway functional enrichment analysis of DE mRNA showed that biological processes were significantly enriched in the chemokine-mediated signaling pathway, immune response, inflammatory response, cell adhesion, and type I interferon signaling pathway. These biological processes have been reported relating to the pathogenesis of pSS [11]. Type I interferon signaling has been found in the pathogenesis of many rheumatic diseases, as well as in pSS. Type I interferon signature genes (ISGs), such as IRF5 and STAT4 [18, 19], have been considered as susceptibility factors in pSS. In patients with pSS, peripheral blood type 1 interferon signature correlates with the existing anti-SSA/Ro antibodies, higher B cell-activating factor (BAFF) gene expression in monocytes, and clinical disease activity [20, 21]. IFI44 and IFI44L, two molecules of ISGs, have been identified as two of the hob genes that may be involved in the development of pSS [12, 13]. In this study, several ISGs, including IFIT1B, IFI27, IFI44, IFI44L and IFI6, were found to be significantly up-regulated in the PBMCs of pSS patients, which suggested the activation of type 1 interferon signaling in these patients.

Another significantly up-regulated gene in this study was EPSTI1. Recent studies have recognized elevated EPSTI1 levels as a promoter for B cell activation by activating TLR9 signaling and considered it a key factor for pSS pathogenesis [14]. In the present study, through the analysis of the PPI network of DE mRNAs, we identified several hub genes, including IL6, IL10, and CXCL8, which are important genes in autoimmune diseases and inflammatory pathways. Based on these findings, we believe that there is an active autoimmune inflammatory response in patients with $\mathrm{pSS}$. 
Table 4 The Top 30 of significant GO enrichment (biological process, molecular function, and cellular component) of differential expressed mRNAs

\begin{tabular}{|c|c|c|c|c|}
\hline GO enrichment & Fold enrichment & Gene number & $P$ value & GO domain \\
\hline cellular ion homeostasis & 18.832 & 3 & 0.009 & Biological process \\
\hline branching involved in labyrinthine layer morphogenesis & 13.950 & 4 & 0.002 & \\
\hline response to yeast & 12.072 & 5 & 0.001 & \\
\hline activation of transmembrane receptor protein tyrosine kinase activity & 10.462 & 4 & 0.006 & \\
\hline labyrinthine layer blood vessel development & 8.260 & 5 & 0.003 & \\
\hline positive regulation of cytokine production & 7.847 & 6 & 0.001 & \\
\hline negative regulation of viral genome replication & 7.062 & 9 & $<0.001$ & \\
\hline chemokine-mediated signaling pathway & 6.631 & 15 & $<0.001$ & \\
\hline regulation of angiogenesis & 6.075 & 6 & 0.003 & \\
\hline positive regulation of peptidyl-threonine phosphorylation & 5.812 & 5 & 0.010 & \\
\hline defense response to fungus & 5.812 & 5 & 0.010 & \\
\hline response to cold & 5.231 & 6 & 0.005 & \\
\hline cell maturation & 5.231 & 6 & 0.005 & \\
\hline cellular defense response & 5.062 & 10 & $<0.001$ & \\
\hline positive regulation of angiogenesis & 4.913 & 18 & $<0.001$ & \\
\hline calcium-mediated signaling & 4.308 & 7 & 0.005 & \\
\hline wound healing & 3.923 & 10 & 0.001 & \\
\hline type I interferon signaling pathway & 3.923 & 8 & 0.004 & \\
\hline positive regulation of protein kinase $B$ signaling & 3.737 & 10 & 0.001 & \\
\hline cellular response to interleukin-1 & 3.537 & 8 & 0.007 & \\
\hline immune response & 2.908 & 39 & $<0.001$ & \\
\hline defense response to virus & 2.853 & 15 & 0.001 & \\
\hline chemotaxis & 2.830 & 11 & 0.006 & \\
\hline azurophil granule & 11.667 & 4 & 0.004 & Cellular component \\
\hline collagen trimer & 3.487 & 10 & 0.002 & \\
\hline chemokine receptor activity & 9.350 & 5 & 0.002 & Molecular function \\
\hline growth factor binding & 5.887 & 5 & 0.010 & \\
\hline chemokine activity & 5.190 & 8 & 0.001 & \\
\hline heparin binding & 3.576 & 18 & $<0.001$ & \\
\hline receptor activity & 2.930 & 20 & $<0.001$ & \\
\hline
\end{tabular}

Table 5 Significantly enriched pathways of differential expressed mRNAs

\begin{tabular}{llll}
\hline Kegg pathway enrichment & Fold enrichment & Gene number & P value \\
\hline Malaria & 4.044 & 26 & $1.14 \mathrm{E}-06$ \\
Cytokine-cytokine receptor interaction & 3.029 & 15 & 0.002 \\
Protein digestion and absorption & 2.574 & 16 & 0.002 \\
Transcriptional misregulation in cancer & 2.543 & 7 & 0.007 \\
Chemokine signaling pathway & 2.435 & 10 & 0.028 \\
Natural killer cell mediated cytotoxicity & 2.320 & 20 & 0.034 \\
Rap1 signaling pathway & 1.887 & 8 & 0.034 \\
Pl3K-Akt signaling pathway & 1.641 & & 0.035 \\
\hline
\end{tabular}




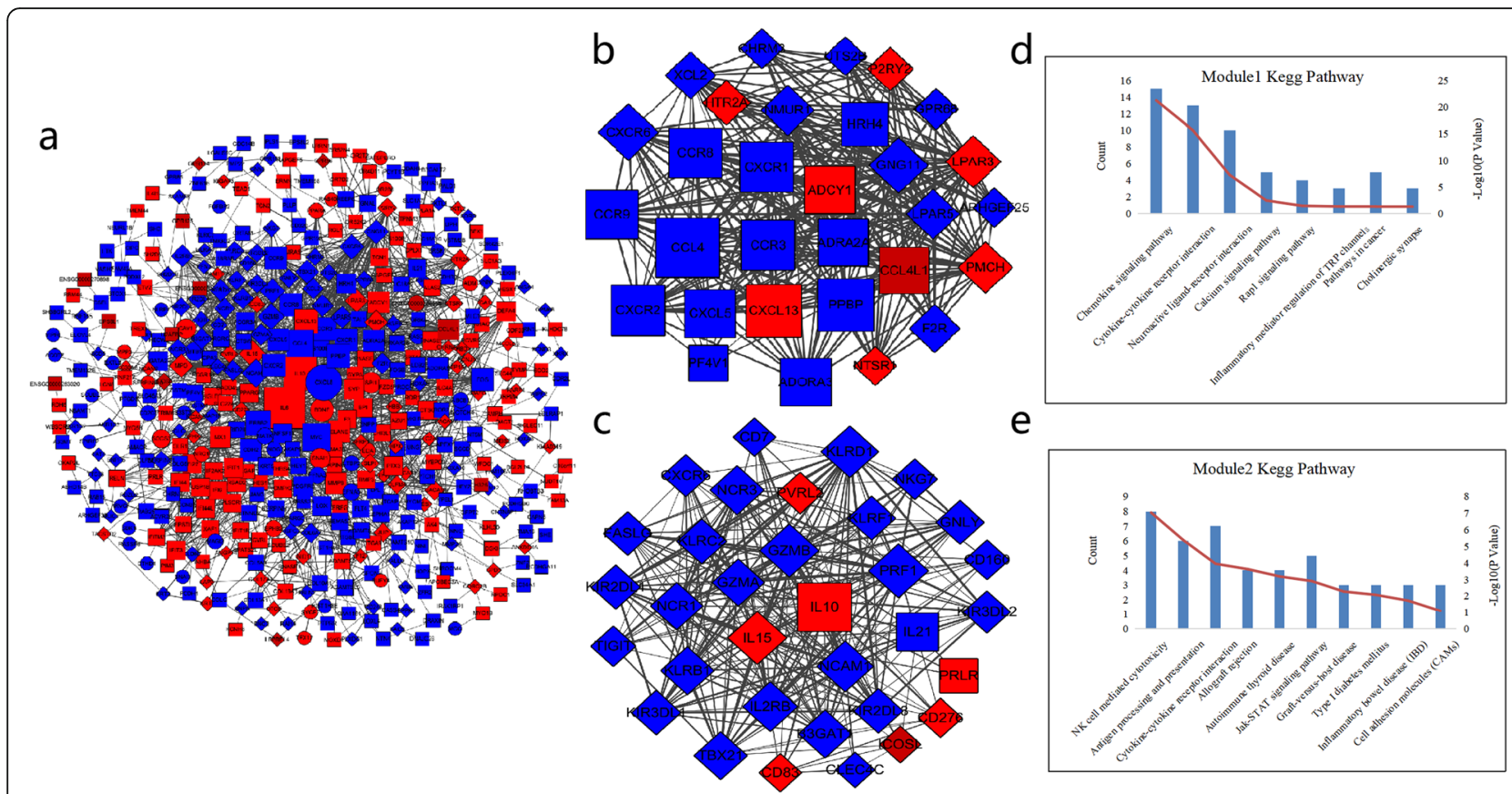

Fig. 2 PPI network of DE mRNAs. (a) PPI network of 640 DE mRNAs, (b) functional module 1, (c) functional module 2. (d) Pathways identification in module 1 by KEGG pathway. (e) Pathways identification in module 2 by KEGG pathway. Red: up-regulated gene; blue: down-regulated gene. Rectangle: cluster gene; ellipse: outlier gene; diamond: overlap gene. The width of lines represents the combined_score of the two interacted proteins

From the top 15 up-regulated and down-regulated DE lncRNAs, we chose four lncRNAs: GABPB1-AS1, PSMA3-AS1, LINC00847 and SNHG1, and constructed their ceRNA network. Further analysis showed that the expression level of two up-regulated lncRNAs (GABPB1-AS1 and PSMA3-AS1) were positively correlated with ISGs (IFI44, IFI44L, IFI6), as well as EPSTI1. These two lncRNAs were validated using real-time PCR in an independent cohort that included 30 pSS patients and 15 healthy controls. The results indicated that GABPB1-AS1 and PSMA3-AS1 were significantly upregulated in patients with pSS. LncRNA GABPB1-AS1

Table 6 Top10 in the result of PPI network connectivity analysis

\begin{tabular}{lll}
\hline Gene & Type & Degree \\
\hline IL6 & Up & 90 \\
CXCL8 & Down & 73 \\
IL10 & Up & 64 \\
NCAM1 & Down & 49 \\
MYC & Down & 46 \\
IL15 & Up & 45 \\
CCL4 & Down & 43 \\
GZMB & Down & 42 \\
PRF1 & Down & 38 \\
CXCR6 & Down & 38 \\
\hline
\end{tabular}

$\overline{P P I}$ protein interaction relationship has been shown to play an important role in cancer pathogenesis through a mechanism that controls gene expression. For example, GABPB1-AS1 is an lncRNA associated with autophagy and may play a key role in glioma biology [22]. GABPB1-AS1 was significantly upregulated in HPV16-positive cervical cancer tissues and associated with a poor prognosis in these patients [23]. It has been shown that GABPB1-AS1 regulates cellular oxidative stress in liver tumor cells and may be an attractive therapeutic target for hepatocellular carcinoma [24]. GABPB1-AS1 inhibited renal cell carcinoma growth and played a tumor suppressor role [25]. Therefore, we predicted that GABPB1-AS1 might participate in the pathogenesis of pSS by affecting type 1 interferon signaling.

BAFF was discovered to be essential in pSS pathogenesis by promoting the activation and proliferation of $\mathrm{B}$ cells and was reported to be significantly up-regulated in PMBCs of pSS patients [26, 27]. According to the prediction in the StarBase database, GABPB1-AS1 interacts with miR-16-5p and miR-155-5p. MiR-16 has been shown to be involved in the occurrence of several autoimmune diseases, as well as in pSS, by controlling cytokine expression [28]. miR-155 is an important regulator of $B$ cell activation through the TNF- $\alpha / B A F F / C D 19$ signaling pathway $[29,30]$. However, miRNAs can act as a "sponge" by lncRNAs to achieve regulatory functions. Therefore, we deduced that GABPB1-AS1 may regulate 

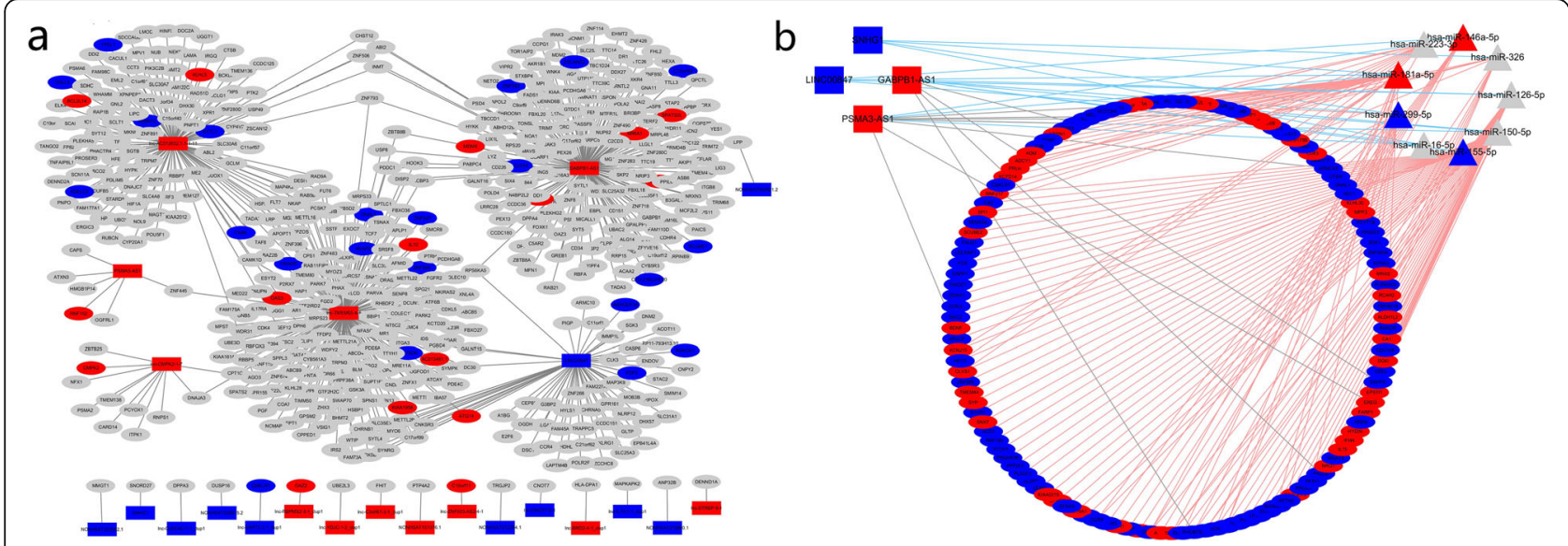

Fig. 3 CeRNA network of selected IncRNAs. (a) The regulation network of the top 15 up-regulation and down-regulation DE IncRNAs predicted by Trans and Cis regulation. (b) CeRNA network of four key IncRNAs based on starBase. Red: up-regulated gene; blue: down-regulated gene. Rectangle: IncRNA; ellipse: mRNA; triangle: miRNA. Grey line: IncRNA-mRNA interaction; red line: miRNA-mRNA interaction; blue line: IncRNA-miRNA interaction

Table 7 Co-expression analysis between 4 key IncRNAs and top15 significantly differentially expressed mRNAs

\begin{tabular}{|c|c|c|c|c|}
\hline LncRNA name & Regulation & Correlated mRNA name & Pearson $r$ & $P$ value \\
\hline \multirow[t]{10}{*}{ GABPB1-AS1 } & Up & SIGLEC1 & 0.803 & 0.016 \\
\hline & & IFI44L & 0.726 & 0.018 \\
\hline & & $|F| 44$ & 0.789 & 0.007 \\
\hline & & $|F| 6$ & 0.890 & 0.001 \\
\hline & & PCNP & 0.880 & 0.004 \\
\hline & & EPSTI1 & 0.835 & 0.003 \\
\hline & & CD83 & 0.739 & 0.036 \\
\hline & & SPI1 & 0.728 & 0.041 \\
\hline & & PLSCR1 & 0.823 & 0.012 \\
\hline & & LAP3 & 0.749 & 0.032 \\
\hline \multirow[t]{5}{*}{ PSMA3-AS1 } & Up & $|F| 44$ & 0.712 & 0.021 \\
\hline & & EPSTI1 & 0.729 & 0.017 \\
\hline & & CD83 & 0.767 & 0.026 \\
\hline & & ZNF267 & 0.854 & 0.007 \\
\hline & & U2AF1 & 0.902 & 0.002 \\
\hline \multirow[t]{5}{*}{ LINC00847 } & Down & MYOM2 & 0.746 & 0.033 \\
\hline & & $\mathrm{SH} 2 \mathrm{D} 1 \mathrm{~B}$ & 0.739 & 0.036 \\
\hline & & KIR2DL3 & 0.756 & 0.030 \\
\hline & & ARMCX3 & 0.838 & 0.010 \\
\hline & & CPA3 & 0.787 & 0.021 \\
\hline \multirow[t]{8}{*}{ SNHG1 } & Down & MYOM2 & 0.902 & 0.002 \\
\hline & & COL6A2 & 0.951 & $<0.001$ \\
\hline & & KIR2DL3 & 0.810 & 0.015 \\
\hline & & ARMCX3 & 0.846 & 0.008 \\
\hline & & APEX1 & 0.840 & 0.009 \\
\hline & & CPA3 & 0.808 & 0.015 \\
\hline & & IL2RB & 0.761 & 0.028 \\
\hline & & LDLRAP1 & 0.833 & 0.010 \\
\hline
\end{tabular}




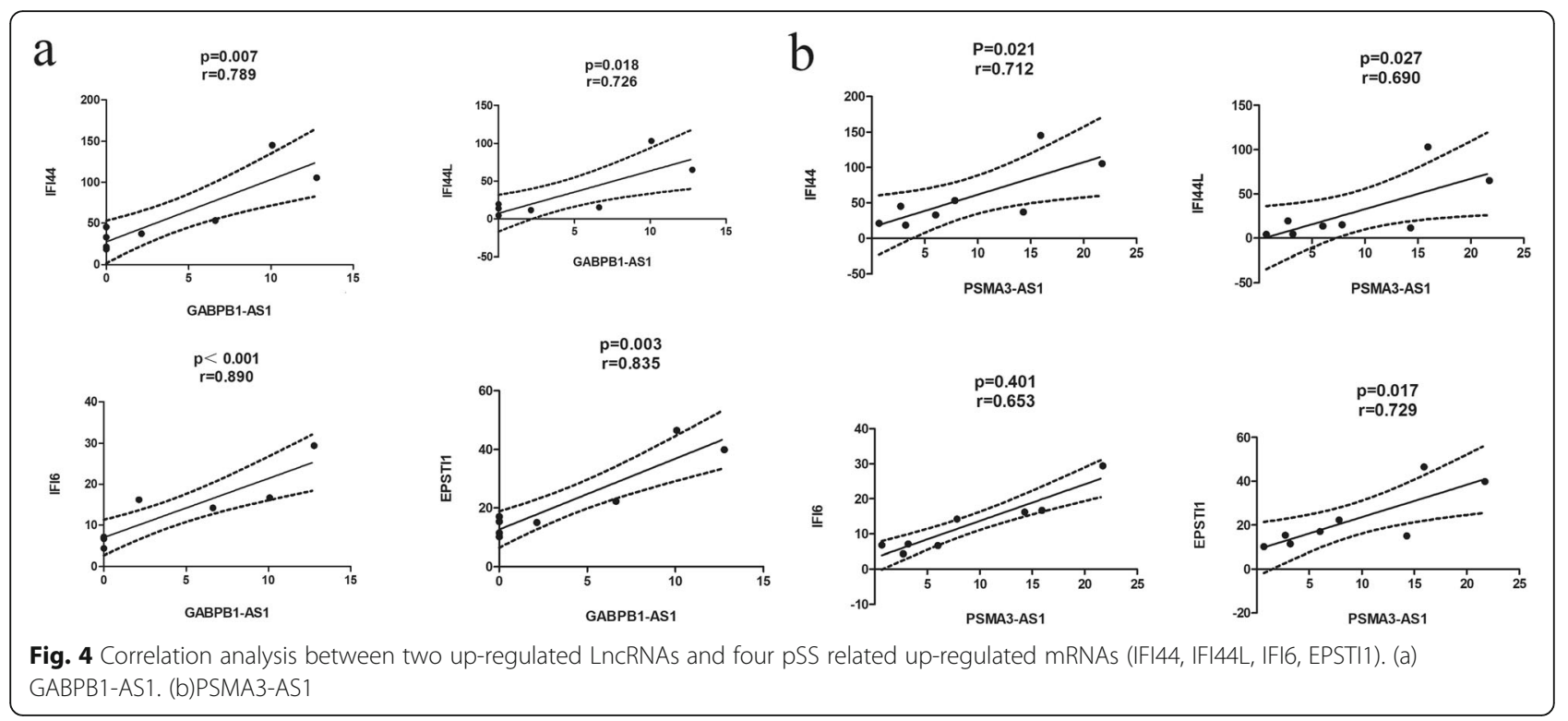

the activation of $\mathrm{B}$ cells by interacting with miR-16 or miR-155. However, this regulatory relationship or other potential regulatory mechanisms underlying differentially expressed lncRNAs affecting pSS pathogenesis needs further experimental verification.
The lncRNA and mRNA expression profile in this study was quite different from the 2 previous similar studies $[10,11]$. Considering that gene expression analysis only reflect a certain stage of the disease, samples from different pSS patients may vary greatly. In this
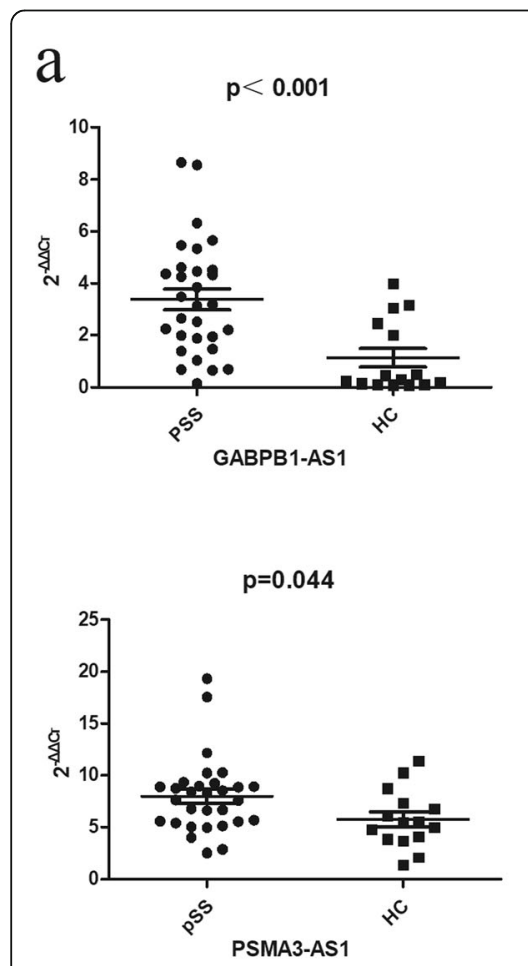

b

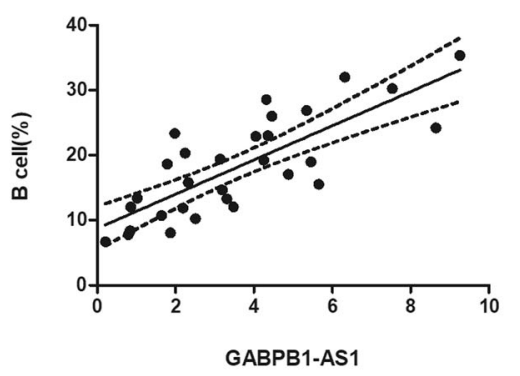

$p=0.009$

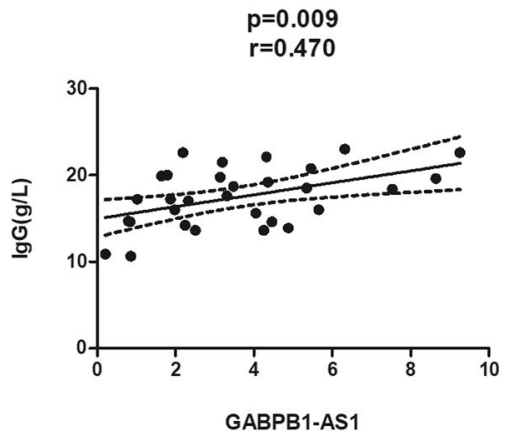

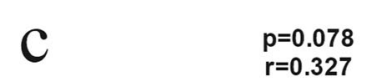
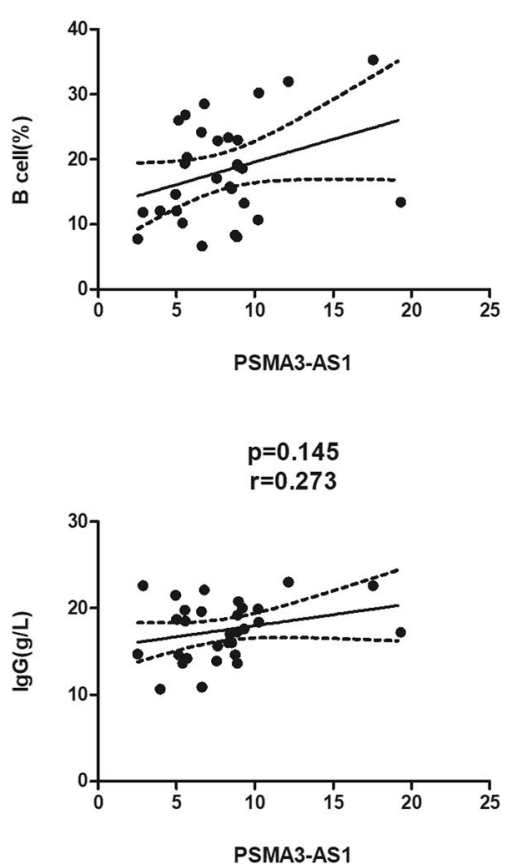

Fig. 5 Correlation analysis between GABPB1-AS1 and PSMA3-AS1 and pathological factors. (a) GABPB1-AS1 and PSMA3-AS1 expression levels in pSS patients $(n=30)$ and healthy persons $(n=15)$ were determined using real-time PCR. (b) Correlation analysis between GABPB1-AS1 and B cell and lgG levels in pSS patients and health controls. (c) Correlation analysis between PSMA3-AS1 and B cell and lgG levels in pSS patients and health controls. The percentage of CD19+ B cells was detected by flow cytometry and IgG was measured by ELISA. The significant difference was $p<0.05$ 
study, the four patients in the dicovery cohort had very similar clinical features. All the pSS patients we recruited hadn't been given immunosuppressive treatment previously and $80 \%$ patients were diagnosed pSS with the result of labial salivary gland biopsies. Compared of $11 \mathrm{DE}$ LncRNAs abtained in previous studies, only NRIR was similarly up-regulated (fold change $=7.1, p=0.002$ ) in this study based on RNA sequencing and other 10 lncRNAs showed no differential expression (Table S4). However, there is some overlap in the expression pattern of gene pathways. Type I interferon signaling pathway was significantly enriched in all 3 studies, indicating its importance in the pathogenesis of pSS. We used realtime PCR to verify the two significantly up-regulated lncRNAs in a relatively large cohort to ensure the reliability of the results. Interestingly, we found the expression level of GABPB-AS1 was significantly positively correlated with the percentage of $\mathrm{B}$ cells. The possible mechanism of GABPB-AS1 involved in the activation of $B$ cell was further analyzed, which laid foundation for the next research.

\section{Conclusion}

In this study, we identified two significantly up-regulated lncRNAs, GABPB1-AS1 and PSMA3-AS1, in pSS patients compared to health controls. GABPB1-AS1 was found to be up-regulated 3.0-fold in pSS patients, and its expression level is positively correlated with the percentage of B cells and IgG levels. GABPB1-AS1 is predicted to play an important role in the pathogenesis of pSS and may be a promising biological marker in the disease.

This study add new evidences on the importance of lncRNAs in the pathogenesis of pSS and may offer promising clues for novel diagnosis method and therapeutic target to the disease.

\section{Methods \\ Patients}

Thirty female patients diagnosed with pSS and 15 sexand age-matched healthy persons were recruited from the Second Affiliated Hospital of Zhejiang University. The enrolled pSS patients met the diagnostic criteria of the 2016 American College of Rheumatology (ACR)European League against Rheumatism (EULAR). The severity of dryness was evaluated using the patientreported visual analogue scale (VAS). Results of laboratory tests and salivary gland biopsy were collected, as well as systemic involvement of the patients. The disease activity of pSS patients was evaluated using ESSDAI. The enrolled patients did not have immunosuppressive treatment prior to blood specimen collection. Peripheral venous blood samples $(5 \mathrm{~mL})$ were extracted from each participant using BD Vacutainer K2EDTA tubes, and PBMCs were immediately isolated by Ficoll-Hypaque density gradient separation. Trizol was added to PBMC samples immediately, then cells were lysed and subjected to total RNA extraction. This mixture was stored in a $80^{\circ} \mathrm{C}$ refrigerator until RNA extraction. The discovery cohort was composed of 4 pSS patients and 4 healthy controls, and the independent validation cohort was composed of $30 \mathrm{pSS}$ patients and 15 healthy controls.

Prior to the blood sample collection, written informed consent was obtained from the participants. The protocols used in this study were performed following the principles of the Helsinki declaration and were approved by the Ethical Committee.

\section{RNA extraction and purification}

Total RNA was extrated from PBMCs using the miRNeasy Mini Kit (Qiagen, Germany) following the manufacturer's instructions. High-purity RNA was obtained using the RNAClean XP Kit (Beckman Coulter, USA) and RNase-Free DNase Set (Qiagen). RIN number of RNA samples was detected on an Agilent Bioanalyzer 2100 (Agilent Technologies, USA) and NanoDrop ND2000 spectrophotometer to evaluate RNA integrity. Samples with qualified measurements (2100 RIN $\geq 7.0,28 \mathrm{~S}$ / $18 \mathrm{~S} \geq 0.7$ ) were included in the subsequent transcriptome sequencing.

\section{Transcriptome sequencing}

Eight RNA samples from the discovery cohort were used for transcrptome sequencing. Sequencing RNA sample library construction was performed through a series of steps, including rRNA removal, fragmenting, first-strand cDNA synthesis, second-strand cDNA synthesis, terminal repair, addition of 3' terminal A overhang, connection, and enrichment. The concentration was determined using a Qubit ${ }^{\circ} 2.0$ Fluorometer and library size on Agilent 4200.

Cluster generation and first-to-sequencing primer hybridization were conducted on the cBot with the Illumina sequencer following the procedures acquired from the cBot instruction. Sequencing reagents were prepared according to the Illumina user guide, and flow cells carrying clusters were used for double-ended sequencing by the paired-end program. Data collection software of Illumina was controlled and analyzed in real-time the sequencing process.

High throughput sequencing (Illumina Hiseq 2000/ 2500 and Miseq) was used to complete the cDNA sequencing. Raw sequencing reads were filtered to obtain clean reads. The spliced mapping algorithm of HISAT2 (version 2.0.4) was used for genome mapping of the clean reads, using a genome version of hg38 (ftp://ftp. ensembl.org/pub/release-90/fasta/homo_sapiens/dna/ Homo_sapiens.GRCh38.dna.toplevel.fa.gz). The number of reads was converted to Fragments Per Kilobase of 
transcript per Million mapped reads (FPKM) values using Perl scripts after fragment counting (StringTie, version 1. 3.0) and normalization (trimmed mean of $M$ values, TMM). Transcriptome sequencing read depths and mapping efficiency was shown in Table S1. Finally, 38,096 lncRNAs and 50,869 mRNAs were investigated.

After eliminating the low-expressed genes in the data (genes whose raw count was 0 in more than $75 \%$ samples), genes with a different expression between pSS samples and health samples were analyzed by edgeR. Meanwhile, we calculated the differential expression multiple (fold-change) based on the FPKM value. LncRNA and mRNA expression with a fold-change $>2$ and a $p$-value less than 0.05 were considered to be differentially expressed genes.

\section{Bioinformatics analysis}

GO and KEGG functional enrichment analysis of mRNAs with different expression levels was accomplished using the DAVID online tool (https://david. ncifcrf.gov/; version 6.8). The results include Biological Process, Cellular Component, and Molecular Function. The Fisher test was used, and the enrichment threshold was set at $p$-value $<0.05$.

The PPI of differentially expressed genes was obtained using the STRING (http://www.string-db.org/; version 10.0) database. PPI networks of differentially expressed genes were constructed using Cytoscape software (version 3.2.0). In the PPI networks, functional modules were identified by the MCODE plug-in of Cytoscape software, and finally, biologically meaningful protein complexes were obtained. Parameters were as follows:include loops: false degree cutoff: 10 , node score cutoff: 0.2, cut: true, FF: false, K-Core: 2 , max. Depth from seed: 100. GO and KEGG pathway enrichment analyses of genes in the modules were carried out.

lncRNA target genes were predicted using Trans and Cis regulation. Cytoscape software was used to construct the regulation network of top 15 up-regulation and down-regulation DE lncRNAs. MiRNAs and mRNAs that have interaction relationship with these $\mathrm{DE}$ IncRNAs were predicted in the StarBase Database (http://starbase.sysu.edu.cn/; version 2.0). The miRNA targets of DE mRNAs were predicted using the MiRDB Database (http://www.mirdb.org/). PSS related miRNAs were obtained from the HMDD Database (http://www. cuilab.cn/hmdd; version 3.2). Combined the interaction relationship acquired from the above two databases and the 21 pSS related miRNAs, the IncRNA-miRNA-mRNA network, or the ceRNA network, was constructed by cytoscape software. Then, the co-expression relationship between the key IncRNAs in the ceRNA network and the 15 most up-regulated and down-regulated $\mathrm{DE}$ mRNAs was analyzed by Spearman's test.

\section{Real-time PCR}

The lncRNAs screened for abnormal expression were validated using real-time PCR in all the 45 extracted RNA samples. The lncRNAs we screened were based on the following criteria: (1) fold change $\geq 4$, (2) average FPKM of the up-regulated groop $\geq 5$, (3) without repeated sequences of mRNA, and (4) lncRNAs without information in databases were excluded.

The purified total RNA was reverse transcribed into cDNA using the ReverTra Ace qPCR Kit (TOYOBO, FSQ-101). The obtained cDNA sample was subjected to real-time quantitative PCR using the Power SYBR Green PCR Master Mix (ABI, USA). A QuantStudio 5 RealTime PCR System (ABI) was used for the PCR procedures. LncRNA expression was measured and normalized to the mean expression of the housekeeping gene: GAPDH (Table S2). The relative fold change of each sample was calculated in relation to the $\Delta \mathrm{Ct}$ of a random unstimulated sample (reference) in the health control group according to the formula: fold change = $2^{-\Delta \Delta \mathrm{Ct}}$, where $\Delta \Delta \mathrm{Ct}=\Delta \mathrm{Ct}$ sample- $\Delta \mathrm{Ct}$ reference.

\section{Statistical analyses}

GraphPad Prism 7.0 version was used for statistical analysis of the data. Data are represented as fold changes relative to healthy controls. Correlation analysis was performed by Spearman's test. An analysis based on the two-tailed unpaired t-test or Mann-Whitney $\mathrm{U}$ test was performed to evaluate differential expression of genes between groups. The significant difference was the $p$ value of less than 0.05 . False discovery correction was not used to adjust $p$-value because of the small number of DE lncRNAs and mRNAs.

\section{Abbreviations \\ pSS: Primary Sjögren's syndrome; IncRNA: Long non-coding RNA: PBMC: Peripheral blood mononuclear cell; PPI: Protein interaction relationship; ceRNA: Competitive endogenous RNA; miRNA: Short-chain non- coding RNA; IFN- $\gamma$ : Interferon- $\gamma$; MMP: Promoting matrix metalloproteinase; ESSDAl: Eular Sjogren's syndrome disease activity index; DE: Differential expression; ISG: Interferon signature gene; BAFF: B cell-activating factor; ACR: American College of Rheumatology; EULAR: European League against Rheumatism; VAS: Visual analogue scale; FPKM: Fragments Per Kilobase of transcript per Million mapped reads}

\section{Supplementary Information}

The online version contains supplementary material available at https://doi. org/10.1186/s12865-021-00439-3.

Additional file 1: Table S1. Transcriptome sequencing read depths and mapping efficiency; Table S2. The selected IncRNAs and the

housekeeping gene (GAPDH) and their primers. Tables S3. Correlation analysis between expression levels of GABPB1-AS1 and PSMA3-AS1 and some clinical parameters. Table S4. Comparison of differentially expressed LncRNAs abtained in previous similar studies with this study. 


\section{Authors' contributions}

XC carried out the molecular genetic studies, participated in the sequence alignment, and drafted the manuscript. QC carried out the molecular genetic studies and performed the statistical analysis. YD helped to collected tissue samples and revise the manuscript. LL participated in the design of the study. HW conceived of the study, and participated in its design and coordination, and helped to revise the manuscript. All authors read and approved the final manuscript.

\section{Funding}

This work is supported by National Natural Science Foundation of China (grant numbers 82071810), Key research and development projects of Zhejiang science and Technology Department (grant number 2020C03044).

\section{Availability of data and materials}

The datasets generated and analysed during the current study are available in the GEO database, https://www.ncbi.n/m.nih.gov/geo/query/acc.cgi?acc= GSE164885.

\section{Declarations}

\section{Ethics approval and consent to participate}

The study was conducted according to the guidelines of the Declaration of Helsinki, and approved by the Ethics Committee of the Second Affiliated Hospital of Zhe Jiang University School of Medicine (2020-433,2020-06-04). Written informed consent has been obtained from all participants prior to the study.

\section{Consent for publication}

Not applicable.

\section{Competing interests}

The authors declare no conflict of interest.

Received: 20 April 2021 Accepted: 13 July 2021

Published online: 20 July 2021

\section{References}

1. Salvi V, Gianello V, Tiberio L, Sozzani S, Bosisio D. Cytokine targeting by miRNAs in autoimmune diseases. Front Immunol. 2019;10:15. https://doi. org/10.3389/fimmu.2019.00015.

2. Long H, Wang X, Chen Y, Wang L, Zhao M, Lu Q. Dysregulation of microRNAs in autoimmune diseases: pathogenesis, biomarkers and potential therapeutic targets. Cancer Lett. 2018;428:90-103. https://doi.org/1 0.1016/j.canlet.2018.04.016

3. Cha S, Mona M, Lee KE, Kim DH, Han K. MicroRNAs in autoimmune Sjogren's syndrome. Genomics Inform. 2018;16(4):e19. https://doi.org/10. 5808/GI.2018.16.4.e19.

4. Mathy NW, Chen XM. Long non-coding RNAs (IncRNAs) and their transcriptional control of inflammatory responses. J Biol Chem. 2017;292(30): 12375-82. https://doi.org/10.1074/jbc.R116.760884.

5. Gomez JA, Wapinski OL, Yang YW, Bureau JF, Gopinath S, Monack DM, et al. The NeST long ncRNA controls microbial susceptibility and epigenetic activation of the interferon-gamma locus. Cell. 2013;152(4):743-54. https:// doi.org/10.1016/j.cell.2013.01.015.

6. Zhang F, Wu L, Qian J, Qu B, Xia S, la T, et al. Identification of the long noncoding RNA NEAT1 as a novel inflammatory regulator acting through MAPK pathway in human lupus. J Autoimmun. 2016;75:96-104. https://doi. org/10.1016/j.jaut.2016.07.012

7. Zhang HJ, Wei QF, Wang SJ, Zhang HJ, Zhang XY, Geng Q, et al. LncRNA HOTAIR alleviates rheumatoid arthritis by targeting miR-138 and inactivating NF-kappaB pathway. Int Immunopharmacol. 2017;50:283-90. https://doi. org/10.1016/j.intimp.2017.06.021.

8. Lu Y, Liu X, Xie M, Liu M, Ye M, Li M, et al. The NF-kappaB-responsive Long noncoding RNA FIRRE regulates posttranscriptional regulation of inflammatory gene expression through interacting with hnRNPU. J Immunol. 2017;199(10):3571-82. https://doi.org/10.4049/jimmunol.1700091.

9. Shi X, Cui Z, Liu X, Wu S, Wu Y, Fang F, et al. LncRNA FIRRE is activated by MYC and promotes the development of diffuse large B-cell lymphoma via Wnt/beta-catenin signaling pathway. Biochem Biophys Res Commun. 2019; 510(4):594-600. https://doi.org/10.1016/j.bbrc.2019.01.105.
10. Peng $Y$, Luo $X$, Chen $Y$, Peng L, Deng C, Fei $Y$, et al. LncRNA and mRNA expression profile of peripheral blood mononuclear cells in primary Sjogren's syndrome patients. Sci Rep. 2020;10(1):19629. https://doi.org/10.1 038/s41598-020-76701-2.

11. Dolcino M, Tinazzi E, Vitali C, et al. Long Non-Coding RNAs Modulate Sjogren's Syndrome Associated Gene Expression and Are Involved in the Pathogenesis of the Disease. J Clin Med. J Clin Med. 2019;8(9):1349. https:// doi.org/10.3390/jcm8091349.

12. Yao Q, Song Z, Wang B, Qin Q, Zhang JA. Identifying key genes and functionally enriched pathways in Sjogren's syndrome by weighted gene co-expression network analysis. Front Genet. 2019;10:1142. https://doi.org/1 0.3389/fgene.2019.01142.

13. Khuder SA, Al-Hashimi I, Mutgi AB, et al. Identification of potential genomic biomarkers for Sjogren's syndrome using data pooling of gene expression microarrays. Rheumatol Int. 2015;35(5):829-36. https://doi.org/10.1007/s002 96-014-3152-6.

14. Sun JL, Zhang HZ, Liu SY, Lian CF, Chen ZL, Shao TH, et al. Elevated EPSTI promote B cell hyperactivation through NF-kappaB signalling in patients with primary Sjogren's syndrome. Ann Rheum Dis. 2020;79(4):518-24. https://doi.org/10.1136/annrheumdis-2019-216428.

15. Sandhya P. Joshi KScaria V Long noncoding RNAs could be potential key players in the pathophysiology of Sjogren's syndrome. Int J Rheum Dis. 2015;18(8):898-905. https://doi.org/10.1111/1756-185X.12752.

16. Shi H, Cao N, Pu Y, Xie L, Zheng L, Yu C. Long non-coding RNA expression profile in minor salivary gland of primary Sjogren's syndrome. Arthritis Res Ther. 2016;18(1):109. https://doi.org/10.1186/s13075-016-1005-2.

17. Wang J, Peng H, Tian J, Ma J, Tang $X$, Rui $K$, et al. Upregulation of long noncoding RNA TMEVPG1 enhances T helper type 1 cell response in patients with Sjogren syndrome. Immunol Res. 2016;64(2):489-96. https:// doi.org/10.1007/s12026-015-8715-4.

18. Lessard CJ, Li H, Adrianto I, et al. Variants at multiple loci implicated in both innate and adaptive immune responses are associated with Sjogren's syndrome. Nat Genet. 2013;45(11):1284-92. https://doi.org/10.1038/ng.2792.

19. Gestermann N, Mekinian A, Comets E, Loiseau P, Puechal X, Hachulla E, et al. STAT4 is a confirmed genetic risk factor for Sjogren's syndrome and could be involved in type 1 interferon pathway signaling. Genes Immun. 2010;11(5):432-8. https://doi.org/10.1038/gene.2010.29.

20. Brkic Z, Maria NI, van Helden-Meeuwsen CG, van de Merwe JP, van Daele PL, Dalm VA, et al. Prevalence of interferon type I signature in CD14 monocytes of patients with Sjogren's syndrome and association with disease activity and BAFF gene expression. Ann Rheum Dis. 2013;72(5):72835. https://doi.org/10.1136/annrheumdis-2012-201381.

21. Maria NI, Brkic Z, Waris M, van Helden-Meeuwsen CG, Heezen K, van de Merwe JP, et al. MxA as a clinically applicable biomarker for identifying systemic interferon type I in primary Sjogren's syndrome. Ann Rheum Dis. 2014;73(6):1052-9. https://doi.org/10.1136/annrheumdis-2012-202552.

22. Luan F, Chen W, Chen M, Yan J, Chen H, Yu H, et al. An autophagy-related long non-coding RNA signature for glioma. FEBS Open Bio. 2019;9(4):65367. https://doi.org/10.1002/2211-5463.12601.

23. Ou R, Lv M, Liu X, Lv J, Zhao J, Zhao Y, et al. HPV16 E6 oncoprotein-induced upregulation of IncRNA GABPB1-AS1 facilitates cervical cancer progression by regulating miR-519e-5p/Notch2 axis. FASEB J. 2020;34(10):13211-23. https://doi.org/10.1096/fj.202000762R

24. Qi W, Li Z, Xia L, Dai J, Zhang Q, Wu C, et al. LncRNA GABPB1-AS1 and GABPB1 regulate oxidative stress during erastin-induced ferroptosis in HepG2 hepatocellular carcinoma cells. Sci Rep. 2019;9(1):16185. https://doi. org/10.1038/s41598-019-52837-8.

25. Gao S, Zhang F, Sun H, Yang X. LncRNA GA-binding protein transcription factor subunit Beta-1 antisense RNA 1 inhibits renal carcinoma growth through an MiR-1246/Phosphoenolpyruvate Carboxykinase 1 pathway. Onco Targets Ther. 2020;13:6827-36. https://doi.org/10.2147/OTT.S257275.

26. Thompson N, Isenberg DA, Jury EC, Ciurtin C. Exploring BAFF: its expression, receptors and contribution to the immunopathogenesis of Sjogren's syndrome. Rheumatology (Oxford). 2016;55(9):1548-55. https://doi.org/10.1 093/rheumatology/kev420.

27. Yoshimoto K, Tanaka M, Kojima M, Setoyama Y, Kameda H, Suzuki K, et al. Regulatory mechanisms for the production of BAFF and IL-6 are impaired in monocytes of patients of primary Sjogren's syndrome. Arthritis Res Ther. 2011;13(5):R170. https://doi.org/10.1186/ar3493.

28. Yan L, Liang M, Hou X, Zhang Y, Zhang H, Guo Z, et al. The role of microRNA-16 in the pathogenesis of autoimmune diseases: a 
comprehensive review. Biomed Pharmacother. 2019;112:108583. https://doi. org/10.1016/j.biopha.2019.01.044

29. Aboelenein HR, Hamza MT, Marzouk H, Youness RA, Rahmoon M, Salah S, et al. Reduction of CD19 autoimmunity marker on B cells of paediatric SLE patients through repressing PU.1/TNF-alpha/BAFF axis pathway by miR-155. Growth Factors. 2017;35(2-3):49-60. https://doi.org/10.1080/08977194.201 7.1345900.

30. Alivernini S, Kurowska-Stolarska M, Tolusso B, et al. MicroRNA-155 influences B-cell function through PU.1 in rheumatoid arthritis. Nat Commun. 2016;7: 12970.

\section{Publisher's Note}

Springer Nature remains neutral with regard to jurisdictional claims in published maps and institutional affiliations.

Ready to submit your research? Choose BMC and benefit from:

- fast, convenient online submission

- thorough peer review by experienced researchers in your field

- rapid publication on acceptance

- support for research data, including large and complex data types

- gold Open Access which fosters wider collaboration and increased citations

- maximum visibility for your research: over $100 \mathrm{M}$ website views per year

At $\mathrm{BMC}$, research is always in progress.

Learn more biomedcentral.com/submissions 